\title{
Tratamento fisioterapêutico tardio em indivíduo submetido a cirurgia de fixação de fêmur, pós-fratura: um estudo de caso
}

\author{
Late physiotherapeutic treatment in individual submitted to fixing surgery femoral, post-fracture: a \\ case study
}

\author{
Letícia Salete do Prado Ferreira $^{1}$, , Andrey Rogério Campos Golias ${ }^{2} \bullet$
}

${ }^{1}$ Fisioterapeuta pelo Centro Universitário Ingá (Uningá), Maringá, Paraná, Brasil. ²Professor Adjunto e supervisor de estágio no Centro Universitário Ingá (Uningá), Maringá, Paraná, Brasil. Autor para correspondência. E-mail: leticiaspradoferreira@outlook.com

\begin{abstract}
Resumo: Introdução: Fraturas proximais de fêmur são comuns após uma queda, sendo consideradas um problema de saúde pública. $\mathrm{O}$ objetivo do estudo foi verificar os efeitos do tratamento fisioterapêutico tardio aplicado em um indivíduo submetido a cirurgia de fixação após fratura de fêmur. Estudo de Caso: Trata-se de um estudo de caso de um paciente de 55 anos, sexo feminino, com fixação de fêmur pós fratura transtrocanteriana de fêmur direito, sob tratamento fisioterapêutico realizado em 12 semanas, o qual envolveu terapias manuais, treino de força, equilíbrio e propriocepção, bem como recursos hidroterapêuticos. Discussão: Após aplicação do tratamento, foi possível observar melhora e manutenção da força muscular e amplitude de movimento, melhora do equilíbrio, propriocepção e redução do quadro álgico. Assim, pode-se concluir que tal abordagem aplicada para a paciente em questão, foi capaz de surtir efeitos positivos relacionados a dor, força muscular, amplitude de movimento, equilíbrio e propriocepção.
\end{abstract}

Palavras-chave: fisioterapia, fraturas do fêmur, hidroterapia, reabilitação.

\begin{abstract}
Introduction: Proximal femur fractures are common after a fall, and are considered a public health problem. The aim of the study was to verify the effects of late physical therapy treatment applied to an individual who underwent fixation surgery after fracture of the femur. Case report: This is a case study of a 55-year-old female patient with femur fixation after trans trochanteric fracture of the right femur, under physical therapy treatment performed in 12 weeks, which involved manual therapies, strength training, balance and proprioception, as well as hydrotherapeutic resources. Discussion: After treatment application, it was possible to observe improvement and maintenance of muscle strength and range of motion, improvement of balance, proprioception and reduction of pain. Thus, it can be concluded that such an approach applied to the patient in question, was able to have positive effects related to pain, muscle strength, range of motion, balance and proprioception.
\end{abstract}

Keywords: physical therapy specialty, femoral fractures, hydrotherapy, rehabilitation.

\section{Introdução}

Durante a vida o ser humano está sujeito a situações que podem acometer sua autonomia e independência funcional. Dentre estas ocorrências destacam-se as quedas, cujo evento pode propiciar limitações biopsicossociais e de qualidade de vida significativas, levando até mesmo à morte, sobretudo a população idosa (Ríos et al., 2012). Isso se explica pelo fato de que, com o passar dos anos, ocorre um declínio gradual em diversas funções de órgãos e sistemas, culminando, por exemplo, em alterações das funções nervosas, sensoriais, cardiovasculares, osteomusculares e controle de equilíbrio, que somado a influência dos fatores ambientais torna o indivíduo suscetível a queda, podendo dessa forma, alavancar os riscos do desenvolvimento de fraturas (Bento et al., 2011).

As fraturas são consideradas lesões traumáticas associadas a interrupção completa ou parcial da continuidade óssea e podem ser categorizadas de acordo com uma gama classificatória, a qual abrange os tópicos relacionados ao contato do foco da fratura com o meio exterior, podendo ser do tipo fechada ou aberta/exposta; gravidade da exposição para as fraturas consideradas expostas; mecanismo de produção da fratura, sendo eles, traumatismos diretos ou indiretos; quanto à presença de fator predisponente a fratura, sendo patológica ou traumática; quanto à localização no sentido longitudinal do osso; ao tipo de desvio; e quanto ao traço da fratura. A finalidade da classificação é atribuída a orientação do tratamento, determinação do prognóstico e importante parâmetro na verificação de determinada abordagem terapêutica aplicada (Ruaro, 2004). 
Entre aquelas ocasionadas por quedas, as fraturas proximais de fêmur correspondem a 1\% das quedas. Esta pode ser classificada em intra-capsular (acometem o colo femoral), ou extra-capsular (transtrocanterianas). A ocorrência das fraturas de fêmur tem aumentado significativamente nos últimos anos, bem como os custos gerados e aumento das internações hospitalares, tornando-se um sério problema de saúde pública e um contribuinte expressivo ao desenvolvimento de morbi-mortalidades (Zago et al., 2009).

O tratamento mais realizado nas fraturas proximais de fêmur é o cirúrgico. Nele, há redução óssea por meio de placas, parafusos, próteses e demais materiais que propiciam a fixação, sendo a conduta cirúrgica e o tipo de material empregados a partir da clínica do paciente. Para isso, devem ser analisados a presença ou não de doenças de base, saúde óssea, nível de consciência e demais fatores apresentados pelo paciente. Já o tratamento conservador será indicado em situações que existam fraturas incompletas, sem desvios e quando o paciente não apresenta prognóstico para deambulação (Venturato, 2016).

A Fisioterapia por meio de seus recursos desempenha fundamental importância na reabilitação dos pacientes que sofreram fraturas, uma vez que estimula o retorno funcional, atua na prevenção de complicações e efeitos deletérios da imobilidade, tanto do paciente submetido ao tratamento conservador, quanto aquele submetido a intervenção cirúrgica. As condutas de tratamento fisioterapêutico para este quadro estarão voltadas conforme necessidades do paciente, almejando a redução da dor, ressaltando as orientações quanto ao pós-operatório, mobilizações, fortalecimento e flexibilidade muscular, transferências, treino de equilíbrio e deambulação com dispositivo auxiliar de marcha, e prevenção de novas quedas (Bento et al., 2011).

Diante do exposto, o estudo do presente tema motiva-se a partir da percepção da frequência das fraturas de fêmur secundárias a queda e suas repercussões funcionais, socioeconômicas e de qualidade de vida, bem como o resultado do tratamento fisioterapêutico designado a estas circunstâncias. Dessa forma, o estudo de caso em questão tem por objetivo verificar os efeitos do tratamento fisioterapêutico tardio aplicado em um indivíduo submetido a cirurgia de fixação após fratura de fêmur.

\section{Relato de caso}

O estudo foi realizado na Clínica Escola de Fisioterapia do Centro Universitário Ingá, Uningá, de Maringá/PR, em 2018. Tal estabelecimento está inserido ao $8^{\circ}$ Conselho de Fisioterapia e Terapia Ocupacional (Crefito-8) e oferece à população de Maringá e região tratamento fisioterapêutico em diversas especialidades, como Ortopedia e Traumatologia Funcional, Fisioterapia Aquática, Neurofuncional, Dermato-funcional, Saúde da Mulher e Gerontologia, todos na modalidade gratuita, estando este serviço conveniado ao Sistema Único de Saúde (SUS). Os atendimentos são realizados por estagiários pertencentes ao último ano de graduação, sob supervisão de professores especialistas nas respectivas áreas (Golias et al., 2007; Viana et al., 2014).

Paciente 55 anos, sexo feminino, $70 \mathrm{kgs}, 1.58 \mathrm{~cm}$ altura, branca, viúva, autônoma, previamente sedentária, não tabagista, não etilista, diagnosticada com Hipercolesterolemia, Osteoporose, Espondilolistese L4-L5, Espondilólise L5 e Fibromialgia (sob tratamento medicamentoso).

Relata que sofreu queda da própria altura em ambiente domiciliar e procurou serviço médico de urgência onde, após avaliação, recebeu diagnóstico clínico de fratura transtrocanteriana completa de fêmur direito, sendo admitida em hospital onde foi submetida a cirurgia de fixação por meio de haste intramedular do tipo Proximal Femoral Nail - PFN (Figura 1). O período de hospitalização foi de 6 dias. A busca por tratamento fisioterapêutico aconteceu somente 6 meses após o trauma, com queixa principal de claudicação, dor em região lateral e anterior de quadril e coxa direita, desequilíbrios, dificuldade em aclives e ao agachar; e impossibilidade de realizar movimentos rápidos com o membro inferior direito (MID).

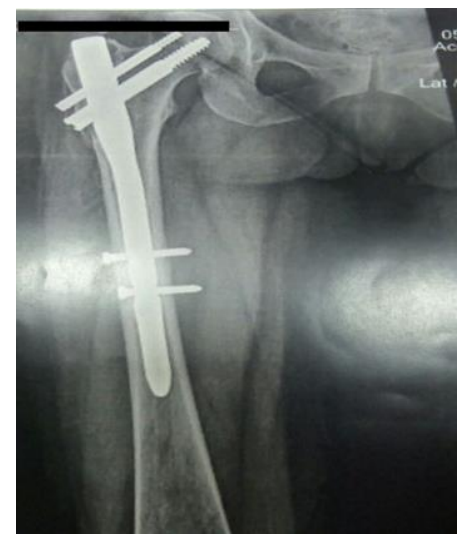

Figura 1. Radiografia coxofemoral direita: controle de evolução de fratura transtrocanteriana do fêmur direito, fixada com haste intramedular PFN. 


\section{Procedimento para coleta dos dados}

Foi realizada entrevista quando as informações pertinentes ao caso foram coletadas, bem como foi realizada avaliação fisioterapêutica, a qual abrangeu a palpação, perimetria, avaliação postural, força e flexibilidade muscular, amplitude de movimento, equilíbrio, marcha, testes específicos e Escala Visual Analógica da Dor (EVA).

Ao que se refere a palpação foi aplicada pressão moderada sob as regiões anterior de coxa e virilha, extensão glútea e região lateral de quadril e coxa, todos do MID, com a paciente posicionada em decúbito dorsal, ventral e lateral esquerdo sob a maca, respectivamente, com região de quadris e membros inferiores (MMII) despidas.

O trofismo muscular foi avaliado por meio da perimetria (Tabela 1), a qual foi realizada nas regiões de coxa, com referência na base da patela, verificando nas distâncias de $5 \mathrm{~cm}, 10 \mathrm{~cm}$ e $15 \mathrm{~cm}$, acima da referência, bilateral; e de panturrilha, com referência no ápice da patela, sentido caudal, sendo as distâncias idênticas às supracitadas, bilateral.

A avaliação postural foi realizada com a paciente somente de roupas íntimas, em ortostatismo, nas posições anterior e posterior, lateral direita e esquerda, de forma relaxada e sem sapatos. O teste de força muscular, foi realizado de forma manual e isométrica, dos flexores, extensores, rotadores internos e externos, adutores e abdutores de quadril; extensores e flexores de joelho; e flexores e extensores de tronco (fibras superiores e inferiores), seguindo a avaliação de força muscular descrita por Kendall et al. (1995) onde, a graduação da força varia em uma progressão de 0 a 5 , sendo grau 0 a ausência de esboço de contração muscular; grau 1 quando há esboço de contração, sem movimento; grau 2 ocorre contração fraca, com movimento a partir do isolamento da gravidade; grau 3 ocorre movimento contra a ação da gravidade, porém sem resistência adicional; grau 4 há contração contra a gravidade e resistência imposta moderadamente; e grau 5 há contração muscular contra resistência superior ao grau anterior.

A avaliação de flexibilidade/encurtamento muscular foi realizada de forma passiva, nos músculos extensores e adutores do quadril. Neste estudo, especialmente para avaliação de encurtamento dos músculos flexores, abdutores, rotadores laterais de quadril, flexores e extensores de joelho, foram realizados os testes de Thomas, Ober, Teste do Piriforme, Sinal do Tripé, e Teste de Ely, respectivamente. A Amplitude de Movimento (ADM) foi realizada por meio da Goniometria, com uso de goniômetro universal, aos movimentos das articulações de quadril e joelho, bilaterais (Kendall, et al., 1995; Magee, 2002).

O equilíbrio dinâmico e semidinâmico foi avaliado por meio do Teste de Equilíbrio de Romberg/Romberg Sensibilizado e Star Excursion Balance Test (SEBT), respectivamente. Durante a aplicação do teste de Romberg e Romberg Sensibilizado, a paciente foi convidada a permanecer em posição ortostática e, após isso, em posição de tandem, respectivamente, sob superfície estável, descalça, com braços ao longo do corpo e oclusão visual, durante 30 segundos cronometrados, para ambas posturas, de modo a verificar as possíveis oscilações durante este tempo (Magee, 2002).

Para aplicação do SEBT foi utilizado fita adesiva, folha sulfite, papel do tipo contact transparente e fita métrica. Dessa forma, a fita adesiva foi dividida em 8 linhas de $120 \mathrm{~cm}$ cada, as quais foram coladas ao chão em formato de estrela, com ângulo de $45^{\circ}$ de distância entre cada reta, sendo que, em cada uma foram identificadas as seguintes direções: anterior (A), ântero-medial (AM), ântero-lateral (AL), lateral (L), medial (M), póstero-lateral (PL), posterior (P) e póstero-medial (PM) (Figura 2), conforme proposto por Artioli et al. (2010).

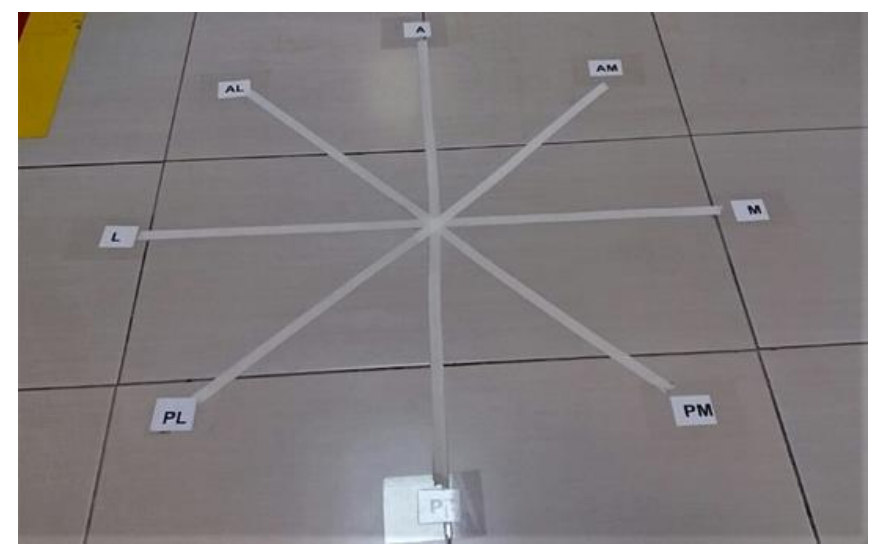

Figura 2. Disposição do SEBT. 
Após preparação dos materiais, a paciente foi orientada sobre o procedimento, e então, convidada a permanecer descalça, em apoio unipodal exatamente ao meio da estrela, enquanto o membro de alcance realizava o máximo deslocamento nas 8 direções em sentido horário, sendo que, no momento em que o deslocamento ocorria, a paciente deveria tocar a fita com os dedos do pé, para que dessa forma fosse possível demarcar posteriormente por meio da fita métrica a distância do alcance obtido. Além disso, foi cronometrado o tempo de execução de ambos testes, tanto com MID em suporte, quanto com o MIE nesta mesma posição. A cronometragem do tempo não está descrita nos protocolos do SEBT conforme as literaturas estudadas, porém no presente estudo, tal variável foi aplicada com o intuito de verificar e comparar a agilidade dos membros durante a avaliação e reavaliação.

A avaliação da marcha ocorreu por meio de observação qualitativa, enquanto a paciente deambulava em linha reta, sob superfície plana e estável com boa luminosidade, descalça, em uma distância média de 4 metros. Para rastreio de demais afecções foram aplicados em ambos membros os testes de Trendelenburg, Patrick-Fabére e Teste de Balanço Pélvico (Magee, 2002).

Em todas sessões a paciente foi questionada sobre sua dor de modo a classificá-la por meio da Escala Visual Analógica (EVA), com o intuito de monitorar o nível álgico, orientar a intervenção e visualizar a evolução ou não do quadro.

\section{Intervenção fisioterapêutica}

As sessões aconteceram no período de fevereiro a abril de 2018, totalizando 16 atendimentos, realizados 2 vezes na semana, com duração média de 40 minutos cada. Dos 16 atendimentos, o primeiro foi designado a avaliação, e o último a reavaliação fisioterapêutica.

Do $2^{\circ}$ ao $5^{\circ}$ atendimento, foram aplicados recursos da terapia manual, sendo eles: deslizamento profundo na direção centrípeta, com pressão moderada e velocidade lenta constante; Pompage Muscular nos tempos de tensionamento do tecido até o limite apresentado, tempo de manutenção e por último, o tempo de retorno conforme velocidade do tecido; e Liberação Miofascial de acordo com o limite da expansibilidade do tecido associado a alongamento muscular; todos durante tempo médio de 30 minutos, nas regiões anterior e lateral de quadril, coxa, e virilha do MID, por meio do uso de óleo de girassol hipoalergênico, com a paciente posicionada em decúbito dorsal e lateral esquerdo, de maneira relaxada. Os 10 minutos finais de cada sessão, foram destinados a aplicação de alongamentos passivos da cadeia anterior, lateral e posterior de tronco e membros inferiores, mantidos por 30 segundos cada, associados a inspirações nasais profundas e expirações orais lentas e prolongadas.

Do $6^{\circ}$ ao $10^{\circ}$ atendimento foram instituídos o aquecimento geral ativo de baixa intensidade por meio de esteira e/ou bicicleta ergométrica, realizado durante 5 minutos; exercícios para fortalecimento muscular, por meio de recursos da cinesioterapia e mecanoterapia, sendo realizados de maneira ativa livre, com progressão para ativa-resistida, de modo isotônico e/ou isométrico, aplicados aos músculos flexores, extensores, abdutores, adutores, e rotadores de quadril; flexores e extensores de joelho; dorsiflexores e plantiflexores de tornozelo; e flexores e extensores de tronco, durante 3 séries de 10 repetições cada, durante 25 minutos em média. Foi repetida a conduta para alongamento muscular conforme sessões anteriores, durante 5 minutos; e aplicado liberação miofascial da região anterior e lateral de coxa a direita em MID aos 5 minutos finais.

Os $11^{\circ}$ e $12^{\circ}$ atendimentos foram realizados Hidroterapia em piscina aquecida com temperatura média de $34^{\circ} \mathrm{C}$. Antes da imersão, foi aferida Pressão Arterial (PA) da paciente, para maior seguridade do tratamento. Na sequência, foram realizados alongamentos ativo-assistidos na posição ortostática, dos músculos da cadeia anterior, posterior e lateral de MMII e tronco, durante 30 segundos cada, durante 5 minutos; seguido por aquecimento do tipo caminhada em velocidade média-rápida pela piscina, durante 5 minutos; prosseguindo para hidrocinesioterapia com uso de flutuadores e pesos, na posição ortostática, para fortalecimento dos músculos flexores, extensores, abdutores e adutores de quadril e dos flexores e extensores de joelho, por 3 séries de 15 repetições cada, durante 15 minutos; após isso, foram empregados exercícios para estimular equilíbrio e coordenação motora com uso de deslizamento turbulento e através de step, por 10 minutos; e ao final, em posição supina, com estabilização pélvica, foi realizado Bad Ragaz para fortalecimento dos flexores e inclinadores de tronco. 


\section{Discussão}

De acordo com Muniz et al. (2007), a incidência de fraturas proximais de fêmur se destaca no público feminino, chegando a uma proporção de 3:1 em relação aos homens. Isso pode ser explicado pelo alto número de doenças crônicas presentes nesse público, pelas atividades domésticas, por terem menor quantidade de massa magra e músculos quando comparadas a homens da mesma idade. As mulheres sofrem declínio de força mais precocemente que os homens por que atingem o pico de potência muscular antes deles.

No início dos atendimentos a paciente se queixava de dor e incapacidade para atividades funcionais e incômodo com a claudicação e não sentia confiança ao descarregar peso sob o MID. Por volta do $4^{\circ}$ atendimento, ela relatou redução da dor, menos claudicação e capacidade de transferir peso ao membro que antes não conseguia.

Nos achados palpatórios do MID pós intervenção foi encontrado ausência de tensão muscular em região intermédia do músculo retofemoral, ílipsoas e em origem dos adutores de quadril no período pós intervenção, pode sugerir efeito positivo das condutas realizadas, sobretudo aquelas aplicadas na fase inicial do tratamento voltadas a reduzir tensões e favorecer relaxamento muscular por meio das técnicas de terapia manual e alongamentos passivos. Bienfait (1997) explica que as técnicas de terapia manual são capazes de ativar o mecanismo da comporta da dor na medula espinhal, pelo fato de as fricções exercidas sob a pele estimularem as fibras nervosas sensoriais, bem como possibilita a restauração da ADM das articulações de quadril e joelho.

Em contrapartida, ainda foram identificados dor a palpação em região lateral de quadril e coxa no período pós intervenção, porém com EVA reduzida para ambas regiões. Lourenço e Pires (2015) explicam que os tecidos moles circundantes da região do trocânter maior do fêmur estão sujeitos a irritações frequentes pelo fato de apresentar diversas inserções musculares e estruturas sensíveis sujeitas a diversas forças deformantes.

O MID durante a avaliação demonstrou um aumento da circunferência anatômica na região de coxa, o que também pôde ser observado na reavaliação. Na panturrilha as medidas não apresentaram diferenças significativas no período de avaliação e reavaliação. No estudo de Rosinski e Golias (2007) também foram utilizadas as mesmas referências anatômicas deste estudo para região de coxa na avaliação de um paciente em tratamento por fratura de fêmur e os resultados são semelhantes aos do atual estudo, uma vez que o membro afetado manifestou contorno anatômico maior quando comparado ao membro não afetado.

Tabela 1. Resultado da Perimetria no período pré e pós intervenção.

\begin{tabular}{cccccc}
\hline Região & Referência & Avaliação & Reavaliação & Avaliação & Reavaliação \\
\hline & Base patelar & MID & MID & MIE & MIE \\
Coxa Antitumoral & $5 \mathrm{~cm}$ & $45,5 \mathrm{~cm}$ & $46,2 \mathrm{~cm}$ & $45,2 \mathrm{~cm}$ & $45,1 \mathrm{~cm}$ \\
& $10 \mathrm{~cm}$ & $49,0 \mathrm{~cm}$ & $54,2 \mathrm{~cm}$ & $48,7 \mathrm{~cm}$ & $50,3 \mathrm{~cm}$ \\
& $15 \mathrm{~cm}$ & $53,4 \mathrm{~cm}$ & $54,7 \mathrm{~cm}$ & $52,0 \mathrm{~cm}$ & $53,2 \mathrm{~cm}$ \\
\hline \multirow{2}{*}{ Panturrilha } & Ápice patelar & MID & MID & MIE & MIE \\
& $5 \mathrm{~cm}$ & $36,6 \mathrm{~cm}$ & $37,0 \mathrm{~cm}$ & $36,4 \mathrm{~cm}$ & $36,5 \mathrm{~cm}$ \\
& $10 \mathrm{~cm}$ & $39,4 \mathrm{~cm}$ & $39,7 \mathrm{~cm}$ & $40,1 \mathrm{~cm}$ & $40,0 \mathrm{~cm}$ \\
\hline
\end{tabular}

Abreviações: MID (Membro Inferior Direito); MIE (Membro Inferior Eesquerdo); CM (Centímetros).

Sobre o grau de força muscular, com base na Tabela 2, é possível inferir que os exercícios de fortalecimento muscular propostos neste estudo foram capazes de manter o grau de força muscular daqueles verificados com grau 5 no período pré intervenção, e promoveram ganho de força para aqueles verificados com grau inferior a 5 durante a avaliação inicial, exceto aos flexores de tronco fibras superiores que permaneceu com grau 2 e, isso pode ter acontecido em decorrência ao curto tempo em que o tratamento foi ofertado. Acreditamos que um tempo maior e/ou maior número de sessões seriam necessários para contemplar o ganho de força também para esse grupo muscular.

Mesquita et al. (2009) em uma revisão de literatura sobre o presente assunto, concluem que dentre as condutas fisioterapêuticas ao paciente submetido a cirurgias pós-fraturas femorais, o fortalecimento dos músculos dos membros inferiores e de tronco devem ser priorizados para que assim seja possível obter estabilização das intervenções cirúrgicas, favorecendo a marcha, o equilíbrio, as atividades de vida diária e prevenção de novas quedas. 
Tabela 2. Comparação do grau de força muscular entre os membros no período pré e pós intervenção.

\begin{tabular}{ccccccc}
\hline Grupo muscular & Avaliação & Reavaliação & Avaliação & Reavaliação & Av. & Reav. \\
\cline { 5 - 6 } & MID & MID & MIE & MIE & Tronco \\
\hline Flexores de quadril & 5 & 5 & 5 & 5 & \\
Extensores de quadril & 5 & 5 & 5 & 5 & \\
Abdutores de quadril & 4 & 5 & 4 & 5 & \\
Adutores de quadril & 4 & 5 & 5 & 5 & \\
Rot. Externos de quadril & 5 & 5 & 5 & 5 & \\
Rot. Internos de quadril & 5 & 5 & 5 & 5 & \\
Extensores de joelho & 5 & 5 & 5 & 5 & \\
Flexores de joelho & 5 & 5 & 5 & 5 & \\
Flexores de tronco (fibra superior) & & & & & \\
Flexores de tronco (fibra inferior) & & & & & \\
Extensores de tronco & & & & & \\
\end{tabular}

Abreviações: MID (Membro Inferior Direito); MIE (Membro Inferior Eesquerdo).

$\mathrm{Na}$ avaliação foram identificados encurtamentos musculares, que após a intervenção evoluíram com flexibilidade dentro dos parâmetros normais. Entretanto, os flexores de quadril durante a reavaliação apresentaram encurtamento muscular, porém consideravelmente reduzidos quando comparados a avaliação inicial, o que também pode estar relacionado ao ganho de ADM de extensão de quadril, conforme exposto na Tabela 3. Além disso, o encurtamento muscular dos rotadores laterais de quadril verificados na avaliação inicial também foi identificado pós intervenção

As condutas realizadas neste estudo trouxeram resultados satisfatórios ao que diz respeito a ADM das articulações de quadril e joelho, uma vez que nestes movimentos houve aumento e/ou manutenção do grau de ADM, o que demonstra evolução do quadro, quando comparado aos valores obtidos no período de avaliação (Tabela 3).

Tabela 3. Comparação da amplitude de movimento articular antes e após o tratamento.

\begin{tabular}{ccccc}
\hline ADM & Avaliação & Reavaliação & Avaliação. & \multicolumn{2}{c}{ Reavaliação } \\
& MID & MID & MIE & MIE \\
\hline Flexão de quadril & $45^{\circ}$ & $100^{\circ}$ & $60^{\circ}$ & $90^{\circ}$ \\
Extensão de quadril & $5^{\circ}$ & $10^{\circ}$ & $8^{\circ}$ & $10^{\circ}$ \\
Abdução de quadril & $20^{\circ}$ & $40^{\circ}$ & $25^{\circ}$ & $40^{\circ}$ \\
Adução de quadril & $5^{\circ}$ & $10^{\circ}$ & $10^{\circ}$ & $10^{\circ}$ \\
Rot. Medial de quadril & $22^{\circ}$ & $30^{\circ}$ & $25^{\circ}$ & $40^{\circ}$ \\
Rot. Lateral de quadril & $20^{\circ}$ & $22^{\circ}$ & $30^{\circ}$ & $40^{\circ}$ \\
Flexão de joelho & $100^{\circ}$ & $115^{\circ}$ & $110^{\circ}$ & $120^{\circ}$ \\
\hline
\end{tabular}

Abreviações: ADM (Amplitude de Movimento); MID (Membro Inferior Direito); MIE (Membro Inferior Eesquerdo); Rot. (Rotação).

Bento et al. (2011) obtiveram resultados semelhantes para a ADM após aplicação de técnicas de terapia manual, alongamento e fortalecimento muscular em seu estudo sobre abordagem fisioterapêutica em um paciente de pós-operatório de fratura de diáfise de fêmur, indicando que tais condutas podem trazer efeitos positivos para esses pacientes.

Sobre o uso do SEBT, observamos que o tempo de realização do teste diminuiu quando comparado no período de avaliação e reavaliação (Tabela 4), assim como foram observadas melhoras na distância deslocada e, consequentemente o equilíbrio semidinâmico, podendo sugerir que a terapêutica aplicada surtiu efeitos positivos para equilíbrio, coordenação motora e agilidade. Artioli et al. (2011), tal ferramenta tem sido frequentemente utilizada por pesquisadores nos Estados Unidos da América em estudos que envolvam o controle neuromuscular e o equilíbrio para a região de tornozelo, sendo apenas sugerido para região de joelho.

A escolha do SEBT para este estudo explica-se a partir da análise do seu fundamento que consiste em verificar o equilíbrio semidinâmico e controle postural, bem como pelo fato de ser rápido, prático e barato. Com isso, recomenda-se novos estudos que avaliação do equilíbrio semidinâmico de pacientes com acometimento no quadril, utilizando tal ferramenta. 
Tabela 4. Comparação de distância e tempo de ambos membros durante a execução do SEBT dos períodos pré e pós intervenção.

\begin{tabular}{|c|c|c|c|c|c|c|c|c|}
\hline Medida & $\begin{array}{c}\text { Avaliação } \\
\text { MID }\end{array}$ & $\mathbf{T}$ & $\begin{array}{c}\text { Reavaliação } \\
\text { MID }\end{array}$ & $\mathbf{T}$ & $\begin{array}{c}\text { Avaliação } \\
\text { MIE }\end{array}$ & $\mathbf{T}$ & $\begin{array}{c}\text { Reavaliação } \\
\text { MIE }\end{array}$ & $\mathbf{T}$ \\
\hline $\mathrm{A}$ & 54,2 & \multirow{8}{*}{$56.20 \mathrm{~s}$} & 60,0 & \multirow{8}{*}{$41.17 \mathrm{~s}$} & 55,4 & & 60,0 & \multirow{8}{*}{$34.10 \mathrm{~s}$} \\
\hline $\mathrm{AM}$ & 51,5 & & 53,7 & & 59,2 & & 60,0 & \\
\hline M & 30,3 & & 39,8 & & 52,7 & & 60,0 & \\
\hline PM & 47,3 & & 47,5 & & 56,0 & 39.35 & 60,0 & \\
\hline $\mathrm{P}$ & 50,5 & & 44,0 & & 47,5 & $\mathrm{~s}$ & 44,4 & \\
\hline PL & 53,5 & & 60,0 & & 37,9 & & 58,3 & \\
\hline $\mathrm{L}$ & 56,5 & & 60,0 & & 19,6 & & 58,5 & \\
\hline $\mathrm{AL}$ & 56,0 & & 60,0 & & 45,2 & & 58,3 & \\
\hline
\end{tabular}

O Romberg sensibilizado (posição de Tandem) na reavaliação apresentou maior tempo para demonstrar o sinal (Tabela 5), o que significa melhor equilíbrio estático. Tais dados corroboram com o estudo realizado por Rosinski e Golias (2007) sobre os efeitos da Fisioterapia em um paciente com fratura de fêmur, já que eles também obtiveram aumento no tempo de manutenção da postura do teste.

Tabela 5. Romberg/Romberg sensibilizado.

\begin{tabular}{ccc}
\hline Sinal & Avaliação & Reavaliação \\
\hline Romberg & Negativo & Negativo \\
Romberg sensibilizado & Positivo as 7 segundos & Positivo aos 23 segundos \\
\hline
\end{tabular}

Acerca da avaliação qualitativa da marcha pré-intervenção foi observado claudicação considerável, rotação externa do quadril e pé direito na fase de apoio e balanço. Já no período pós-intervenção, foi observada importante redução desses padrões. Assunção et al. (2009) identificou que a presença de comorbidades está atrelada a má qualidade da marcha nos indivíduos, sendo que, aqueles sem doenças ortopédicas ou que adquiriram carga parcial anterior há 30 dias tendem a demonstrar melhor desempenho durante a marcha. Neste estudo não é possível afirmar que a claudicação presente no pré e pós intervenção seja decorrente a discrepância dos membros, por que tal avaliação não foi realizada.

Não foram identificadas instabilidades de quadril e disfunções na articulação coxofemoral e Sacroilíaca (Quadro 1). Segundo Goodman e Snyder (2002), o Fisioterapeuta deve ser capaz de identificar sinais e sintomas além daqueles referidos pelo paciente, relacionados a alterações sistêmicas e estruturais passíveis de simular ou predispor disfunções musculoesqueléticas ou neuromusculares, de modo desempenhar o diagnóstico diferencial e conhecer melhor o quadro do paciente.

Quadro 1. Comparação dos resultados dos testes específicos de ambos membros durante o período pré e pós intervenção.

\begin{tabular}{|c|c|c|c|c|}
\hline \multirow{2}{*}{ Teste } & Avaliação & Reavaliação & Avaliação & Reavaliação \\
\cline { 2 - 5 } & MID & MID & MIE & MIE \\
\hline Trendelenburg & Negativo & Negativo & Negativo & Negativo \\
\hline Patrick (Faber) & Negativo & Negativo & Negativo & Negativo \\
\hline Balanço da Sacroilíaca & Negativo & Negativo & Negativo & Negativo \\
\hline
\end{tabular}

Fonte: a autora

Outro resultado satisfatório foi a evolução do quadro álgico, pressupondo efeito positivo da abordagem empregada (Gráfico 1).

\section{Limitações do estudo}

O presente estudo possui várias limitações que merecem ser explanadas. Primeiro, o número de sessões e o tempo foram considerados pequenos frente ao que se desejava realizar com a paciente. Segundo, não foi avaliado a discrepância dos membros, sendo este um exame fundamental aqueles que foram submetidos a 
cirurgia de fêmur, uma vez que pode explicar ou não a presença de claudicação e suas consequências / compensações.

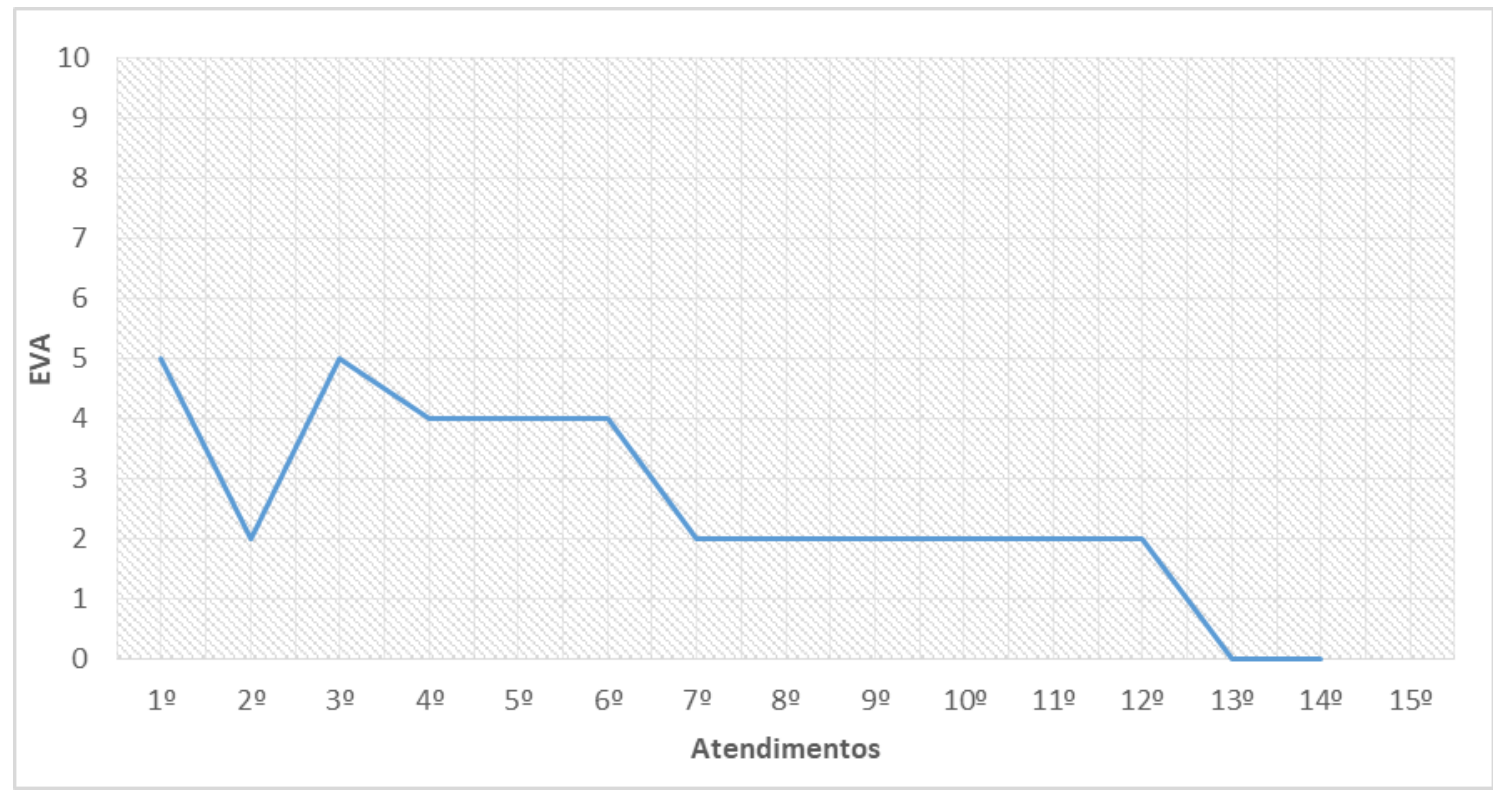

Gráfico 1. Evolução da Escala Visual Analógica da Dor (EVA).

\section{Considerações finais}

O programa de intervenção fisioterapêutica tardia de 12 sessões, proposto para o caso em questão, foi capaz de produzir efeitos positivos ao quadro clínico e queixas relatadas. Por se tratar de um estudo de caso, não é possível se basear apenas pela aplicação de tais condutas e, sugerimos, dessa forma, a realização de estudos com amostra e tempo de tratamento maiores do que os apresentados neste caso, para que assim os resultados sejam mais confiáveis e reprodutíveis. Além disso, o SEBT parece ser uma ferramenta eficaz para avaliar o equilíbrio semidinâmico de pacientes com o quadro clínico semelhante ao do paciente desse estudo.

\section{Referencias}

Artioli, D. P., Portolez, J. L. M. \& Bertolini, G. R. F. 2010. Aplicação do teste de controle neuromuscular em indivíduos com lesão de membro inferior submetidos a tratamento fisioterapêutico. Fiep bulletin, 80.

Assunção, J. H., Fernandes, T. L., Santos, A. L. G., Sakaki, M. H., \& Zumiotti, A. V. 2009. Fatores preditivos para marcha na fratura transtrocanteriana do fêmur. Acta Ortopédica Brasileira, 17(1), 35-39.

Bento, N. T., Vidmar, M. F., Silveira, M. M., \& Wibelinger, L. M. 2011. Intervenções fisioterapêuticas no pósoperatório de fratura de fêmur em idosos. Revista Brasileira de Ciências da Saúde, 9(27), 42-48.

Bienfait, M. 1997. Bases elementares técnicas de terapia manual e osteopatia (2a ed.). São Paulo, SP: Summus.

Golias, A. R. C., Grabowski, J. L., \& Ornellas, E. 2007. Perfil epidemiológico do setor de ortopedia e traumatologia da clínica escola de fisioterapia da Uningá do período de fevereiro a junho de 2007. Revista UNINGÁ, 14(1),179-187.

Goodman, C. C., Snyder, T. E. K. 2002. Diagnóstico diferencial em fisioterapia (4a ed.). Elsevier Virtual Books.

Kendall, F. P., McCreary, E. K., \& Provance, P. G. 1995. Músculos: provas e funções. $4^{\mathrm{a}}$ ed. São Paulo: Manole.

Lourenço, R. B. T., \& Pires, R. E. S. 2015. Fraturas subtrocantéricas do fêmur: atualização. Revista Brasileira de Ortopedia, 51(3), 246-253.

Magee, D. J. 2002. Avaliação musculoesquelética. $3^{\text {a }}$ ed. São Paulo: Manole.

Mesquita, G. V., Lima, M. A. L. T. A., Santos, A. M. R., Alves, E. L. M., Brito, J. N. P. O., \& Carvalho e Martins, M. C. 2009. Morbimortalidade em idosos por fratura proximal do fêmur. Texto \& Contexto - Enfermagem, 18(1), 67-73.

Muniz, C. F., Yoshida, M., Arnaut, A, C., \& Trellha, C. S. 2007. Caracterização dos idosos com fraturas de fêmur proximal atendidos em um hospital escola público. Revista Espaço para a Saúde, 8(2), 33-38. 
Ríos, A. U., Herrera, D. A. C., Ortega, A. N. G. \& Aluma, E. E. P. 2012. Morbilidad y mortalidad en pacientes mayores de 60 años con fractura de cadera en el Hospital Universitario San Vicente Fundación, de Medellín, Colombia. Iatreia, 25(4), 305-313.

Rosinski, T. T., \& Golias, A. R. C. 2007. Hipertrofia muscular em paciente polifraturado de membro inferior: relato de caso. Revista UNINGÁ, 13(1), 13-20.

Ruaro, A. F. 2004. Ortopedia e traumatologia: temas fundamentais e a reabilitação. Umuarama: Elenco.

Venturato, B. B. 2016. Atuação da fisioterapia no pós-operatório da fratura proximal do fêmur em idosos: uma revisão de literatura. 2016. Monografia (Especialização) - Curso de Fisioterapia, Fisioterapia em Geriatria e Gerontologia, Universidade Federal de Minas Gerais, Belo Horizonte, 30p.

Viana, J. R. S., Raiocovitch, T., Golias, A. R., Peternella, F. M. N., Mendes, F. C. V., \& Grabowski, J. 2014. Avaliação da satisfação com a fisioterapia de pacientes atendidos em uma clínica escola de Maringá-PR. Revista UNINGÁ Review, 17(2), 16-21.

Zago, A. P. V., Grasel, C. E. \& Padilha, J. A. 2009. Incidência de atendimentos fisioterapêuticos em vítimas de fraturas em um hospital universitário. Fisioterapia em Movimento, 22(4), 565-573.

\section{Minicurrículo}

Letícia Salete do Prado Ferreira. Fisioterapeuta (2018) pelo Centro Universitário Ingá, Uningá, em Maringá, Paraná.

Andrey Rogério Campos Golias. Doutor em Saúde Coletiva pela Universidade Paulista Julio de Mesquita Filho (2013), Professor Adjunto e supervisor de estágio no Centro Universitário Ingá, Uningá, em Maringá, Paraná.

\footnotetext{
Como citar: Ferreira, L.S.P., \& Golias, A.R.C. 2021. Tratamento fisioterapêutico tardio em indivíduo submetido a cirurgia de fixação de fêmur, pós-fratura: um estudo de caso. Pubsaúde, 7, a118. DOI: https://dx.doi.org/10.31533/pubsaude7.a118

Recebido: 16 dez. 2020.

Revisado e aceito: 18 mai. 2021.

Conflito de interesse: os autores declaram, em relação aos produtos e companhias descritos nesse artigo, não ter interesses associativos, comerciais, de propriedade ou financeiros que representem conflito de interesse.

Licenciamento: Este artigo é publicado na modalidade Acesso Aberto sob a licença Creative Commons Atribuição 4.0 (CC-BY 4.0).
} 